\title{
Determinants of exaggerated difference in morning and evening home blood pressure in Japanese normotensives
}

\author{
Hiroshi Kawabe and Ikuo Saito
}

The aim of this study was to evaluate factors contributing to morning-evening difference in systolic blood pressure (SBP) in Japanese normotensive subjects. A total of 605 Japanese subjects (393 men and 212 women; mean age, 38.7 years) receiving no antihypertensive agents and without diagnosed hypertension ( $\geqslant 140 / 90 \mathrm{~mm} \mathrm{Hg}$ ) were studied using self-measured home BP monitoring. Triplicate morning and evening measurements were performed on 7 consecutive days. The mean of the second and third measurements, as well as the mean of measurements on days 2-7 were adopted as an individuals' home BP value. The morning-evening difference in SBP ranged from -24.8 to $34.8 \mathrm{~mm} \mathrm{Hg}$. The highest quartile (Q4) group of the morning-evening difference ( $\geqslant 4.3 \mathrm{~mm} \mathrm{Hg}, n=151)$ comprised an older age $(43.6 \pm 10.2$ vs. $37.1 \pm 9.8$ years, $P<0.01$ ) and a higher prevalence rate of men (72.8 vs. $62.3 \%, P<0.05)$, regular alcohol drinkers $(45.0$ vs. $24.9 \%, P<0.01)$ and measurement after bathing at night (50.3 vs. $35.2 \%, P<0.01$ ) than did the other quartile groups (Q1-Q3, $n=454)$. In multiple logistic regression analysis, independent risk factors for the Q4 group were older age (10 years older: odds ratio (OR) $1.67, P<0.01,95 \%$ confidence interval (Cl): 1.37-2.04), regular alcohol drinking (OR 1.98, $P<0.01, \mathrm{Cl}: 1.28-3.07$ ) and post-bathing measurement at night (OR 1.97, $P<0.01, \mathrm{Cl}: 1.30-2.97$ ). In conclusion, older age, regular alcohol drinking and post-bathing measurement at night were significant determinants of an exaggerated morning-evening difference in SBP in Japanese normotensives. Hypertension Research (2009) 32, 1028-1031; doi:10.1038/hr.2009.138; published online 4 September 2009

Keywords: alcohol; bathing; home blood pressure; morning-evening difference; older age

\section{INTRODUCTION}

The Japanese Society of Hypertension recommends two measurements of home blood pressure (BP) in a day (that is, after rising in the morning and before going to bed in the evening). ${ }^{1,2} \mathrm{BP}$ is a parameter that readily changes, and its circadian variation is well known. However, if BP is measured under the same regulated conditions, the morning home BP value in normotensive subjects is essentially the same as the evening home BP value; however, numerous experiences of measuring home BP have shown that some subjects exhibit exaggerated differences in morning and evening values. ${ }^{3}$

Recently, we evaluated the effects of drinking alcohol and bathing at night, ${ }^{4,5}$ which are common practices in many Japanese people, on home BP values. We also evaluated the influence of short sleep duration, ${ }^{6}$ which is increasing progressively and causing many health problems in industrialized countries, on home BP values. From our results, these three factors produced different effects on morning and evening home BP values; it was indicated that these factors might be determinants of a difference in morning and evening home BP values. However, we know that many other factors can affect morning and evening home BP; age, sex, current smoking status and medication are the possible candidates. A report that evaluated determinants of an exaggerated difference in morning and evening home BP values in medicated hypertensive patients has been presented, ${ }^{7}$ but no study has evaluated these factors in detail in normotensive subjects. As home BP measurement will be expected to increase in non-hypertensive subjects for the early detection or prevention of hypertension, clarifying these points will become an important issue.

In this study, we evaluated the determinants of an exaggerated difference in morning and evening systolic BP (SBP) in Japanese people with no medication and no diagnosis of hypertension.

\section{METHODS}

Subjects

The subjects were the same as in our previous report. ${ }^{3}$ In brief, a search for candidates was conducted through the intranet of a company, and this study included 700 subjects (468 men and 232 women; mean age, 40.6 years) who were a minimum of 20 years of age (range: $20-84$ years). These subjects provided written consent to participate in this study, and performed measurements according to the protocol. ${ }^{3}$ We then narrowed the subject group to 605 subjects ( 393 men and 212 women; mean age, 38.7 years) who did not take any antihypertensive agents and were not diagnosed as having hypertension $(\geqslant 140 / 90 \mathrm{~mm} \mathrm{Hg})$. Before initiating the study, we collected information 
regarding present illness, history of disease and present smoking status, etc. Shift workers were excluded because their schedules conflicted with the requirements of this study. This study was approved by the Institutional Review Board of the Health Center, Keio University, and the Ethics Committee of the participating company.

\section{BP measurements}

Home BP measurements were performed between 16 October and 13 November 2002. ${ }^{3}$ Using a semiautomatic device (HEM-759P; Omron Life Science, Tokyo, Japan) on the basis of the cuff-oscillometric principle, the subjects were asked to take measurements at the same upper arm position for 7 consecutive days. Self-measured BP was conducted thrice on each occasion with the subjects in a seated position with the arm bare. The measurement was performed after $\geqslant 2$ min of rest. The morning measurement was made within $1 \mathrm{~h}$ after rising, and was carried out before breakfast and after passing urine. When performing measurement before going to bed, the subjects were instructed to avoid measurement within $30 \mathrm{~min}$ after bathing. The subjects were asked to paste all the printed records of BP values on the sheet and submit it. Measurements were started on Tuesday, Wednesday or Thursday. According to the method adopted by our center, the mean of the second and third measurements was taken as the home BP value on one occasion in this study. ${ }^{3}$ The home BP value of an individual was defined as the mean of measurements on days 2-7 (total of 12 BP measurements each in the morning and evening). ${ }^{3}$ To test the validation of the device, a simultaneous measurement using both arms was performed, using the auscultatory method on one side and the testing device on the other. ${ }^{1}$ Each measurement was then repeated using the contralateral arm. In this study, the difference between the auscultatory method and the device was within $5 \mathrm{~mm} \mathrm{Hg}$ in 20 selected subjects.

From the submitted records, we calculated the average of the morning and evening SBP values at home (morning-evening average SBP). The morningevening difference in SBP at home (morning-evening difference in SBP) was defined as morning SBP minus evening SBP.

Casual BP was measured by the patients' own doctors using a mercury sphygmomanometer while the subjects were seated, after at least a 2-min rest. A value measured within 1 month before or after the period of measurement of home BP (including measurements taken at regular health check-ups) was adopted as casual BP.

\section{Evaluation of alcohol drinking, bathing and sleep duration}

During the study period, subjects entered their daily information regarding alcohol drinking, bathing and sleep duration on the sheet. They recorded whether alcohol consumption was done at night, and whether BP measurement in the evening was taken before or after bathing. To avoid measurement within 30 min after bathing, they were instructed to record the time after bathing. They were also instructed to record their sleep duration.

\section{Statistical methods}

Data are shown as mean \pm s.d. Comparisons of two parameters were made by two-tailed non-paired Student's $t$-test, and comparisons of categorical variables were made by the $\chi^{2}$ test. One-way ANOVA (analysis of variance) was performed to detect differences among groups, and Tukey's honestly significant differences test was used for multiple pairwise comparisons of means among groups. Odds ratio (OR) and the $95 \%$ confidence interval $(\mathrm{CI})$ were calculated by multiple logistic regression analysis. Statistical analyses were performed using the computer software SPSS version 11.0 (SPSS, Chicago, IL, USA). A probability value $P<0.05$ was considered statistically significant.

\section{RESULTS}

Background characteristics of subjects

The age of the total study population ranged from 20 to 84 years (mean: $38.7 \pm 10.3$ years), and 393 men and 212 women were enrolled (Table 1). No subjects were taking antihypertensive medication or had previously been diagnosed as having hypertension ( $\geqslant 140 / 90 \mathrm{~mm} \mathrm{Hg})$. Although hyperlipidemia and diabetes mellitus were present in 11.2 and $1.3 \%$ of subjects, respectively, other complicating diseases were rare.

\section{Table 1 Background of subjects}

\begin{tabular}{|c|c|}
\hline Age (years) & $38.7 \pm 10.3$ \\
\hline Male/female & $393 / 212$ \\
\hline Body mass index $\left(\mathrm{kg} \mathrm{m}^{-2}\right)$ & $22.4 \pm 3.0$ \\
\hline Smokers (\%) & 21.1 \\
\hline Regular alcohol drinkers ${ }^{\mathrm{a}}(\%)$ & 29.9 \\
\hline Post-bathing measurement ${ }^{\mathrm{b}}(\%)$ & 39.0 \\
\hline Short sleep duration ${ }^{\mathrm{c}}(\%)$ & 41.3 \\
\hline Hyperlipidemia (\%) & 11.2 \\
\hline Diabetes mellitus (\%) & 1.3 \\
\hline Chronic renal disease (\%) & 0.3 \\
\hline Angina pectoris (\%) & 0.2 \\
\hline Myocardial infarction (\%) & 0.2 \\
\hline Stroke (\%) & 0 \\
\hline
\end{tabular}

a Drinking on $\geqslant 4$ days during 6-day study period.

Measurement after bathing on $\geqslant 4$ days.

Less than $7 \mathrm{~h}$ sleep on $\geqslant 4$ days.

Table 2 Characteristics of highest quartile (Q4) of morning-evening difference in SBP

\begin{tabular}{|c|c|c|c|}
\hline & \multicolumn{3}{|c|}{ Morning-evening difference in SBP } \\
\hline & $\begin{array}{c}\text { Q1-Q3 group } \\
(\mathrm{n}=454) \\
(-24.8-4.1 \mathrm{~mm} \mathrm{Hg})\end{array}$ & $\begin{array}{c}\text { Q4 group } \\
(\mathrm{n}=151) \\
(4.3-34.8 \mathrm{~mm} \mathrm{Hg})\end{array}$ & P-value \\
\hline Age (years) & $37.1 \pm 9.8$ & $43.6 \pm 10.2$ & $<0.01$ \\
\hline Sex (\% men) & 62.3 & 72.8 & 0.02 \\
\hline Body mass index $\left(\mathrm{kg} \mathrm{m}^{-2}\right)$ & $22.3 \pm 3.0$ & $22.7 \pm 2.9$ & NS \\
\hline Smokers (\%) & 22.5 & 17.2 & NS \\
\hline Regular alcohol drinkers ${ }^{a}(\%)$ & 24.9 & 45.0 & $<0.01$ \\
\hline Post-bathing measurement ${ }^{\mathrm{b}}(\%)$ & 35.2 & 50.3 & $<0.01$ \\
\hline Short sleep duration $(\%)$ & 42.1 & 39.1 & NS \\
\hline Casual SBP (mm Hg) & $116.2 \pm 13.4$ & $122.9 \pm 14.4$ & $<0.01$ \\
\hline Casual DBP (mm Hg) & $71.4 \pm 10.5$ & $76.6 \pm 10.3$ & $<0.01$ \\
\hline Morning SBP $(\mathrm{mm} \mathrm{Hg})$ & $113.5 \pm 12.6$ & $124.1 \pm 14.0$ & $<0.01$ \\
\hline Morning DBP (mm Hg) & $71.2 \pm 9.5$ & $78.4 \pm 10.4$ & $<0.01$ \\
\hline Morning HR (per min) & $66.4 \pm 8.5$ & $67.0 \pm 9.2$ & NS \\
\hline Evening SBP (mm Hg) & $115.2 \pm 13.4$ & $114.9 \pm 12.7$ & NS \\
\hline Evening DBP (mm Hg) & $68.7 \pm 9.2$ & $69.8 \pm 9.1$ & NS \\
\hline Evening HR (per min) & $70.4 \pm 9.6$ & $72.3 \pm 10.1$ & 0.04 \\
\hline ME average of SBP $(\mathrm{mm} \mathrm{Hg})$ & $114.4 \pm 12.8$ & $119.5 \pm 13.2$ & $<0.01$ \\
\hline ME average of DBP $(\mathrm{mm} \mathrm{Hg})$ & $70.0 \pm 9.1$ & $74.1 \pm 9.5$ & $<0.01$ \\
\hline ME average of HR (per min) & $68.4 \pm 8.3$ & $69.7 \pm 8.9$ & NS \\
\hline
\end{tabular}

Abbreviations: DBP, diastolic blood pressure; HR, heart rate; ME, morning-evening; NS, not significant; SBP, systolic blood pressure.

Values presented as means \pm s.d.

Drinking on $\geqslant 4$ days during 6 -day study period.

Measurement after bathing on $\geqslant 4$ days.

CLess than $7 \mathrm{~h}$ sleep on $\geqslant 4$ days.

\section{Morning-evening difference in SBP}

The morning-evening difference in SBP ranged from -24.8 to $34.8 \mathrm{~mm} \mathrm{Hg}$ (mean: $1.1 \mathrm{~mm} \mathrm{Hg}$ ), and the highest quartile (Q4) of the morning-evening difference was $\geqslant 4.3 \mathrm{~mm} \mathrm{Hg}(n=151$, median: $7.8 \mathrm{~mm} \mathrm{Hg}$ ).

Determinants of an exaggerated morning-evening difference in SBP We compared the Q4 group of the morning-evening difference with the other three quartile groups $(\mathrm{Q} 1-\mathrm{Q} 3, n=454)$, and evaluated the determinants of an exaggerated morning-evening difference in SBP (Table 2). The subjects in the Q4 group were older and had a higher 
Table 3 Multiple logistic regression analysis for highest quartile (Q4) of morning-evening difference in SBP

\begin{tabular}{|c|c|c|c|}
\hline & \multicolumn{3}{|c|}{ Model } \\
\hline & Odds ratio & P-value & $95 \% \mathrm{Cl}$ \\
\hline Age (10 years) & 1.67 & $<0.01$ & $1.37-2.04$ \\
\hline Male sex & 1.26 & 0.37 & $0.76-2.10$ \\
\hline Smokers & 0.60 & 0.06 & $0.35-1.02$ \\
\hline Regular alcohol drinkers ${ }^{a}$ & 1.98 & $<0.01$ & $1.28-3.07$ \\
\hline Post-bathing measurement ${ }^{b}$ & 1.97 & $<0.01$ & $1.30-2.97$ \\
\hline Short sleep duration ${ }^{c}$ & 0.97 & 0.89 & $0.65-1.46$ \\
\hline ME average of SBP $(10 \mathrm{~mm} \mathrm{Hg})$ & 1.11 & 0.34 & $0.90-1.37$ \\
\hline Casual SBP (10 mm Hg) & 1.13 & 0.21 & $0.93-1.37$ \\
\hline
\end{tabular}

Abbreviations: $\mathrm{Cl}$, confidence interval; $\mathrm{ME}$, morning-evening; SBP, systolic blood pressure.

aDrinking on $\geqslant 4$ days during 6 -day study period.

${ }^{b}$ Measurement after bathing on $\geqslant 4$ days.

'Less than $7 \mathrm{~h}$ sleep on $\geqslant 4$ days.

prevalence of male sex and of 'regular alcohol drinkers' than those in the Q1-Q3 groups. There were more subjects who performed evening home BP measurements after bathing in the Q4 group than in the Q1-Q3 groups, but no difference was found in sleep duration. The prevalence of present smokers tended to be lower in the Q4 group than in the Q1-Q3 groups $(P=0.21)$. There were significant differences in the morning-evening average SBP and in casual SBP between the Q4 group and the Q1-Q3 groups, but no significant difference was found in evening SBP (Table 2).

In multiple logistic regression analysis, the OR (95\% CI) for the Q4 group of the morning-evening difference in SBP was 1.67 (1.37-2.04) for age $(10$-year increase) $(P<0.01), 1.98(1.28-3.07)$ for 'regular alcohol drinkers' $(P<0.01)$ and $1.97(1.30-2.97)$ for 'post-bathing measurement' $(P<0.01)$ compared with the other three quartile groups (Q1-Q3) (Table 3). The OR (95\% CI) for current smokers was $0.60(0.35-1.02)(P=0.06)$ (Table 3$)$.

\section{Relationship between alcohol drinking and taking a bath}

There was little morning-evening difference in SBP in subjects who were not 'regular alcohol drinkers' and who performed evening measurements before bathing $(-0.53 \pm 6.12 \mathrm{~mm} \mathrm{Hg}, \quad n=277)$ (Figure 1). On the other hand, similar morning-evening differences in SBP were found in subjects who were 'regular alcohol drinkers' in the 'pre-bathing measurement' group $(2.45 \pm 6.33 \mathrm{~mm} \mathrm{Hg}, n=92)$ and in subjects who were 'non-regular alcohol drinkers' in the 'postbathing measurement' group $(1.75 \pm 5.75 \mathrm{mmHg}, n=147)$. The morning-evening difference in SBP was the largest in subjects who were 'regular alcohol drinkers' in the 'post-bathing measurement' group $(3.48 \pm 6.43 \mathrm{~mm} \mathrm{Hg}, n=89)$.

The morning-evening average SBP was the lowest in subjects who were 'non-regular alcohol drinkers' in the 'post-bathing measurement' group $(111.0 \pm 12.5 \mathrm{~mm} \mathrm{Hg}, n=147)$, and showed significant differences from that in the other three groups. The highest value of morning-evening average SBP was seen in subjects who were 'regular alcohol drinkers' in the 'pre-bathing measurement' group $(121.0 \pm 11.0 \mathrm{~mm} \mathrm{Hg}, n=92)$, and showed a significant difference from that in the two 'non-regular alcohol drinkers' groups.

\section{DISCUSSION}

In this study, home BP measurement was performed for 7 consecutive days in 605 subjects excluding evident hypertensive patients, and
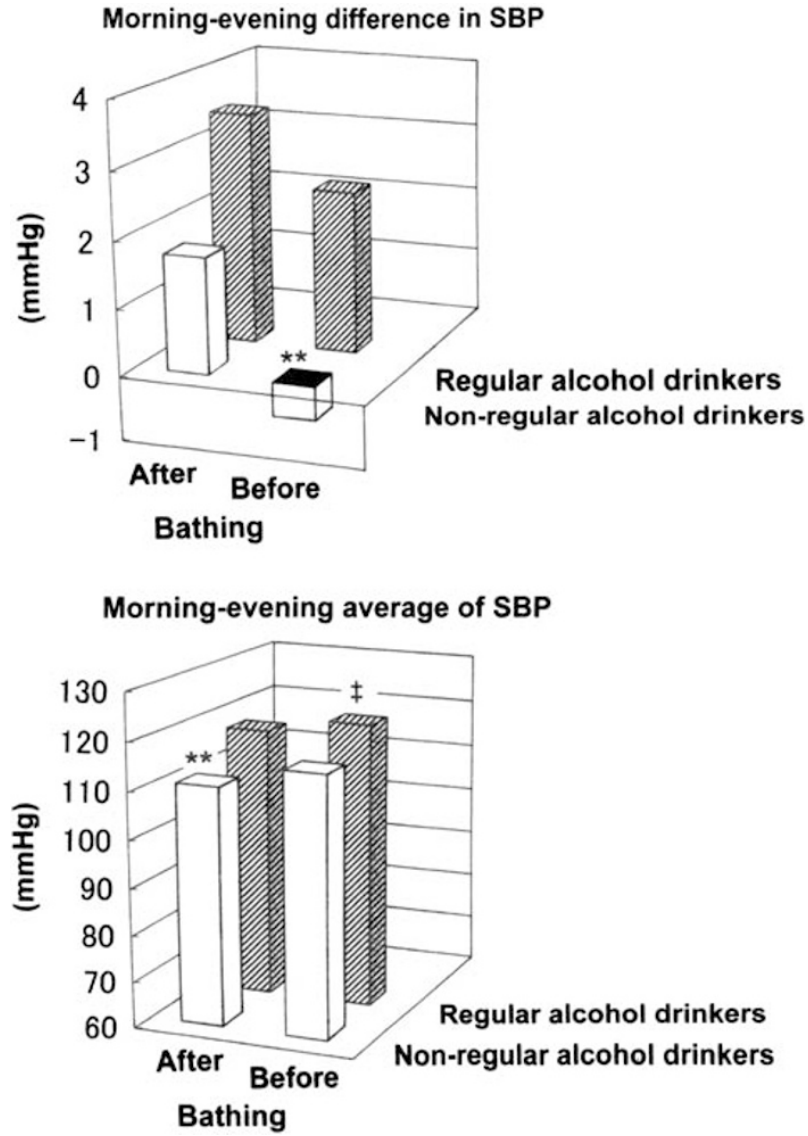

Figure 1 Relationship between effects of drinking alcohol and taking a bath on the morning-evening difference in systolic blood pressure (SBP) and the morning-evening average of SBP. ${ }^{* *} P<0.01$ vs. other three groups, $\ddagger P<0.01$ vs. 'non-regular alcohol drinkers.'

morning and evening values were collected. From analyses of our data, older age, regular alcohol drinking and measurement after bathing at night were recognized as independent determinants of an exaggerated morning-evening difference in SBP.

In this study, we defined 'regular alcohol drinkers' as subjects who consumed alcohol on $\geqslant 4$ days during the 6-day study period, except on the first day. The highest quartile (Q4) group of the morningevening difference in SBP showed a significantly higher prevalence of 'regular alcohol drinkers' than did the other three quartile groups. Kawano et al. ${ }^{8}$ have reported that drinking alcohol produced biphasic effects (decrease in BP at night and increase in BP in the morning) on self-measured home BP values in hypertensive patients who consumed alcohol regularly. However, our recent data on normotensive subjects showed a depressor effect of alcohol drinking at night, but we did not observe an increase in BP in the morning. ${ }^{4}$ In this study, although our comparison was made between 'regular alcohol drinkers' and 'nonregular alcohol drinkers', it showed that drinking alcohol at night produced an increase in the morning-evening difference in SBP. As drinking alcohol at night obviously decreases evening home BP and increases evening heart rate, it is considered that sympathetic nerve activity may be activated by drinking alcohol. Such activated sympathetic nerve activity may produce an increase in the morning home BP value, as suggested by Kawano et al., ${ }^{8}$ and may increase the morningevening difference in SBP. 
With regard to the influence of nighttime bathing on evening home $\mathrm{BP}$ value, we reported that measurements within $1 \mathrm{~h}$ after bathing showed lower values than did those taken before bathing. ${ }^{5}$ We further showed that this depressor effect of bathing disappeared by $1 \mathrm{~h}$ after bathing. ${ }^{5}$ Although we did not evaluate the interval between bathing and measurement in this study, a high frequency of measurement after bathing was a risk factor for an exaggerated morning-evening difference in SBP.

In general, the morning-evening difference in SBP includes two types. One difference is an exaggerated morning SBP elevation, and the other is a large evening SBP reduction. From our previous data, we concluded that Japanese people prefer drinking alcohol and taking a bath at night, and both behaviors have been shown to have a depressor effect. ${ }^{4,5}$ Therefore, these two factors, but not an increase in age, contribute to a decrease in evening home SBP values and consequently increase the morning-evening difference in SBP. This possibility was clarified by the present observation of a relationship between the effects of bathing and drinking alcohol on the morning-evening difference in SBP. We found little morning-evening difference in SBP in subjects who did not drink alcohol and measured evening home BP before bathing. On the other hand, the largest morningevening difference in SBP was observed in subjects who regularly consumed alcohol and measured evening home BP values after bathing. Apart from patients who continuously monitored their home BP values, attention should be paid to diagnosing patients with shortterm home BP monitoring, especially from measurements before going to bed. If we use evening home $\mathrm{BP}$ as morning home $\mathrm{BP}$, we have to teach the subjects to measure evening home BP before bathing and drinking alcohol. If evening home BP is measured after taking a bath, we need to instruct subjects to measure it at least $1 \mathrm{~h}$ after bathing to avoid its depressor effect, as indicated by our recent report..$^{5}$

Although it is known that smoking increases BP and heart rate during the period of smoking, ${ }^{9}$ the relationship between smoking and sustained hypertension is controversial. ${ }^{10,11}$ In this study, the prevalence of present smokers tended to be lower in the highest group of the morning-evening difference in SBP, and smoking itself had a tendency to reduce the risk for an exaggerated morning-evening difference in SBP. This result is the same as that in a recent paper in which determinants of an exaggerated difference in morning and evening $\mathrm{BP}$ values were evaluated in medicated hypertensive patients. ${ }^{7}$ Such a tendency seems to be seen in both hypertensive patients and normotensive subjects. However, the mechanism has not yet been clarified.

Although it is not a changeable factor, increase in age was also a risk factor for an exaggerated morning-evening difference in SBP. It is said that morning BP is usually influenced by $\alpha$-sympathetic nerve activation, ${ }^{12}$ but older people show imbalance of $\alpha_{1}$ - and $\beta$-sympathetic nerve effects. This imbalance may cause increased variability of BP in the elderly. ${ }^{13-15}$ Furthermore, baroreceptor sensitivity has been reported to be decreased in the elderly. ${ }^{16}$ It has been reported that such impaired baroreceptor sensitivity is a key physiological mechanism of the exaggerated morning-evening difference in SBP in relation to the predominant $\alpha$-sympathetic activity in the elderly. ${ }^{17,18}$

In conclusion, there were some cases of an exaggerated morningevening difference in SBP even in normotensive subjects, and determinants of this difference were increase in age, regular alcohol drinking and post-bathing BP measurement at night. Among these determinants, when we evaluate the effect of alcohol drinking in detail, we need to consider various factors such as the amount of alcohol intake on one occasion and the time interval between drinking alcohol and measurement. Therefore, further detailed studies are required to evaluate the above-mentioned determinants.

1 Imai Y, Otsuka K, Kawano Y, Shimada K, Hayashi H, Tochikubo O, Miyakawa M, Fukiyama K, on behalf of the Japanese Society of Hypertension. Japanese Society of Hypertension (JSH) Guidelines for Self-Monitoring of Blood Pressure at Home. Hypertens Res 2003; 26: 771-782.

2 Japanese Society of Hypertension. Japanese Society of Hypertension Guidelines for the Management of Hypertension (JSH 2004). Hypertens Res 2006; 29 (suppl): S1-S105.

3 Kawabe H, Saito I, Saruta T. Influence of repeated measurement on one occasion, on successive days, and on workdays on home blood pressure values. Clin Exp Hypertens 2005; 27: 215-222.

4 Kawabe H, Saito I, Saruta T. Effects of nighttime alcohol intake on evening and next morning home blood pressure in Japanese normotensives. Clin Exp Hypertens 2007; 29: 43-49.

5 Kawabe H, Saito I. Influence of nighttime bathing on evening home blood pressure measurements: how long should the interval be after bathing? Hypertens Res 2006; 29: 129-133.

6 Kawabe H, Saito I. Does short sleep duration in daily life affect morning home blood pressure? Evaluation in Japanese people. Clin Exp Hypertens 2008; 30: 183-190.

7 Ishikawa J, Kario K, Hoshide S, Eguchi K, Morinari M, Kaneda R, Umeda Y, Ishikawa S, Kuroda T, Hojo Y, Shimada K, on behalf of the J-MORE Study Group. Determinants of exaggerated difference in morning and evening blood pressure measured by selfmeasured blood pressure monitoring in medicated hypertensive patients: Jichi Morning Hypertension Research (J-MORE) Study. Am J Hypertens 2005; 18: 958-965.

8 Kawano Y, Pontes CS, Abe H, Takishita S, Omae T. Effects of alcohol consumption and restriction on home blood pressure in hypertensive patients: serial changes in the morning and evening records. Clin Exp Hypertens 2002; 24: 33-39.

9 Minami J, Ishimitsu T, Matsuoka H. Effect of smoking cessation on blood pressure and heart rate variability in habitual smokers. Hypertension 1999; 33: 586-590.

10 Lee D-H, Ha M-H, Kim J-R, Jacobs Jr DR. Effects of smoking cessation on changes in blood pressure and incidence of hypertension. A 4-year follow-up study. Hypertension 2001; 37: 194-198.

11 Mann SJ, James GD, Wang RS, Pickering TG. Elevation of ambulatory systolic blood pressure in hypertensive smokers. A case-control study. JAMA 1991; 265: 2226-2228.

12 Kawano Y, Tochikubo O, Minamisawa K, Miyajima E, Ishii M. Circadian variation of haemodynamics in patients with essential hypertension: comparison between early morning and evening. J Hypertens 1994; 12: 1405-1412.

13 Callister R, Johnson DG, Seals DR. Age and gender influence muscle sympathetic nerve activity at rest in healthy humans. Hypertension 1993; 21: 498-503.

14 Jones PP, Shapiro LF, Keisling GA, Jordan J, Shannon JR, Quaife RA, Seals DR. Altered autonomic support of arterial blood pressure with age in healthy men. Circulation 2001; 104: 2424-2429.

15 Dinenno FA, Dietz NM, Joyner MJ. Aging and forearm postjunctional $\alpha$-adrenergic vasoconstriction in healthy men. Circulation 2002; 106: 1349-1354.

16 Gobbin B, Pickering TG, Sleight P, Peto R. Effect of age and high blood pressure on baroreflex sensitivity in man. Circ Res 1971; 29: 424-431.

17 Tochikubo O, Kawano Y, Miyajima E, Toshihiro N, Ishii M. Circadian variation of hemodynamics and baroreflex functions in patients with essential hypertension. Hypertens Res 1997; 20: 157-166.

18 Jones PP, Chiristou DD, Jordan J, Seals DR. Baroreflex buffering is reduced with age in healthy men. Circulation 2003; 107: 1770-1774. 